\title{
Noise Removal from Multiple MRI Images
}

\author{
Stephen J. Garnier, Griff L. Bilbro, Wesley E. Snyder, and James W. Gault
}

We introduce a novel technique for magnetic resonance image (MRI) restoration, using a physical model (spin equation). We determine a set of three basis images (proton density and nuclear relaxation times) from the MRI data using a nonlinear optimization method, and use those images to obtain restorations of the original image. MRIs depend nonlinearly on proton density, two nuclear relaxation times, $T_{1}$ and $T_{2}$, and two control parameters, echo time (TE) and relaxation time (TR). We model images as Markov random fields and introduce a maximum a posteriori restoration method, based on nonlinear optimization, which reduces noise while preserving resolution.

Copyright $(1994$ by W.B. Saunders Company

KEY WORDS: image analysis, magnetic resonance, noise removal, physical model, basis images, restoration, Markov random fields, optimization.

$\mathbf{T}$ HE SPIN EQUATION' relates the image signal strength measured at a particular pixel, $i$, in image $c$, to three intrinsic properties of a pixel, $P D_{i}, T 1_{i}$, and $T 2_{i}$, and to two measurement parameters, echo time $\left(\mathrm{TE}_{\mathrm{c}}\right)$, and relaxation time $\left(\mathrm{TR}_{\mathrm{c}}\right)$ :

$$
\mathrm{S}_{\mathrm{c}, \mathrm{i}}=\mathrm{PD} \mathrm{D}_{\mathrm{i}} \exp \left(\frac{-\mathrm{TE} \mathrm{E}_{\mathrm{c}}}{\mathrm{T} 2_{\mathrm{i}}}\right)\left(1-\exp \left(\frac{-\mathrm{TR}_{\mathrm{c}}}{\mathrm{T} \mathbf{1}_{\mathrm{i}}}\right)\right) \text {. }
$$

In this report we use equation 1 for simplicity, but our method is suitable to more accurate (and more complex) physical models also. Given a set of such measurements taken at a particular pixel, but with different TE and TR values, one could, in the absence of noise, invert the spin equation and solve for PD, T1, and T2. ${ }^{2}$ These $\mathrm{PD}, \mathrm{T} 1$, and $\mathrm{T} 2$ images can be used to generate images equivalent to data images obtainable with different TE and TR parameters, thereby minimizing the MR system time..$^{2-4}$ Because any TE and TR can be simulated from PD, T1, and $\mathrm{T} 2$, we refer to these three images as "basis" images. However, the inversion problem is ill conditioned unless TE and TR are chosen carefully. For arbitrary (but different) choices of TE and TR, a numerical method must be used for the solution. We develop this method by assuming that there exist three unknown images, $\mathrm{PD}, \mathrm{T} 1$, and $\mathrm{T} 2$. At pixel $\mathrm{i}$, a measure of the difference between a single measurement, $G_{c, i}$, and the physical model from equation 1 , may be written as

$$
H_{c, i}=\frac{1}{2 \sigma_{c}^{2}}\left(S_{c, i}-G_{c, i}\right)^{2}
$$

where $\sigma_{c}$ represents the standard deviation of (assumed) stationary, signal-independent, isotropic uncorrelated additive Gaussian noise ${ }^{5}$ on the $c$ th measurement.

Summing over the measurements and the pixels results in a function

$$
\mathrm{H}_{\mathrm{N}}=\sum_{\mathrm{c}} \sum_{\mathrm{i}} \mathrm{H}_{\mathrm{c}, \mathrm{i}}
$$

that represents the total difference between the measured data and the images resulting from the estimated PD, T1, and T2 values. Thus, if we can then find the values of PD, T1, and T2, which minimize $\mathrm{H}_{\mathrm{N}}$, the "noise-cost" term, we will have a good estimate of these values. Such minimization may be accomplished by gradient descent or other numerical techniques.

Minimizing equation 3 over the PD, T1, T2 domain results in a maximum likelihood (ML) restoration. If one has prior knowledge of some locally homogeneous characteristic of the surfaces of the PD, T1, and T2 images, this knowledge can be applied in a "prior-cost" term, and a maximum a posteriori (MAP) restoration with greater noise reduction can be achieved. We use a measure of the difference between neighboring pixels to remove noise from the $\mathrm{PD}, \mathrm{T} 1$, and $\mathrm{T} 2$ images because we assume that neighboring pixels belonging to the same tissue should appear similar, and should possess similar noise statistics. Our choice for this difference measure is based on our previous

From the Department of Electrical and Computer Engineering, North Carolina State University, Raleigh.

Supported by US Ammy Research Office under Contract No DAAL03-89-D-0003-0004 and by the Center for Communica tions and Signal Processing, North Carolina State University.

Address reprint requests to Griff $L$. Bilbro, PhD, Research Associate Professor, Department of Electrical and Computer Engineering, North Carolina State University, PO Box 7911, Raleigh, NC 27695-7911.

Copyright $\subseteq 1994$ by W.B. Saunders Company

0897-1889/94/0704-0005\$3.00/0 

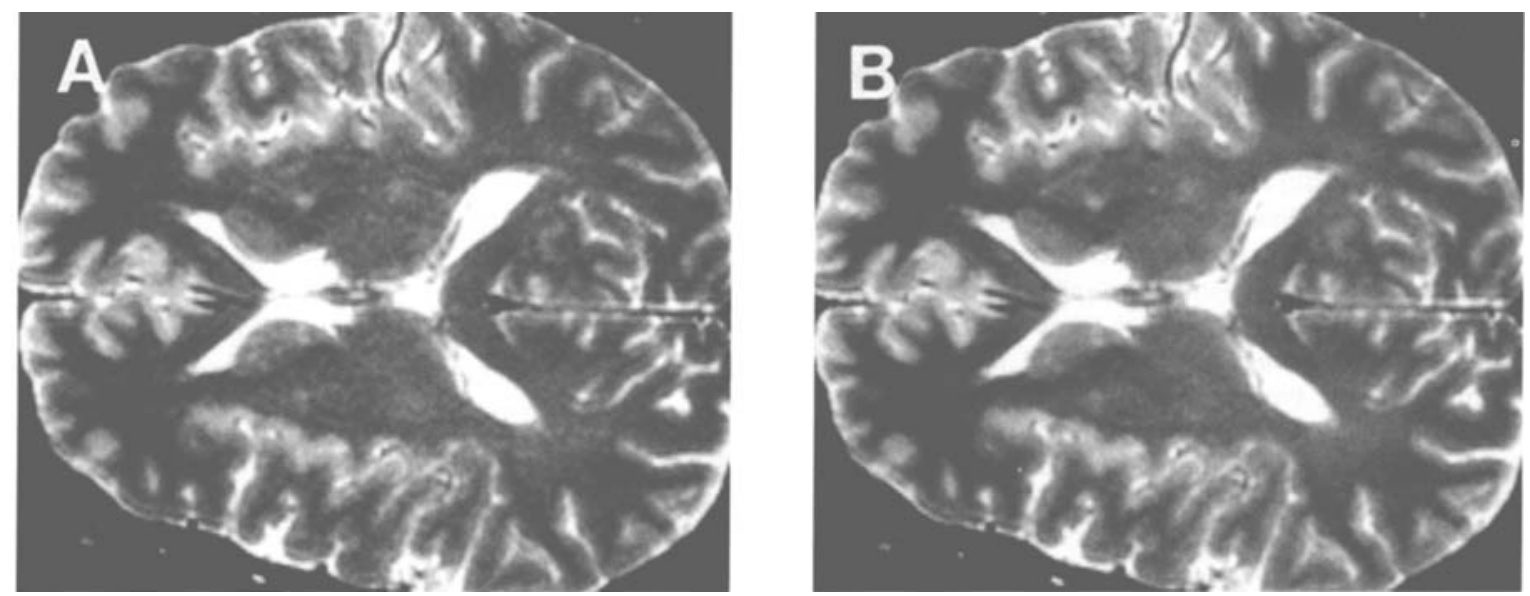

Fig 1. TE, 90; TR, 3,000. Left, Data; Right, MAP restoration. Three millimeter slice with 3-mm spacing.

experience ${ }^{6,7}$ with several approaches towards the nonlinear restoration of images.

For a single pixel of interest, say pixel $i$, and its surrounding neighborhood, $\aleph_{i}$, the prior cost for the $P D$ signal may be represented by $E_{P D, i}$, where

$$
E_{P D, i}=\frac{\sum_{n \in X_{i}}\left(P_{i}-P D_{i+\eta}\right)^{2}}{2 \sigma_{P D}^{2}+\frac{1}{\tau^{2}} \sum_{n \in X_{i}}\left(P D_{i}-P D_{i+\eta}\right)^{2}} .
$$

The value for $\sigma_{\mathrm{PD}}$, a well-chosen estimate of noise in the $\mathrm{PD}$ image, indicates the emphasis of smoothing on the PD image. The parameter $\tau$ is a smoothly changing annealing parameter. At infinite $\tau$, the prior term performs simple linear smoothing; a low-pass filter. As $\tau$ is decreased,

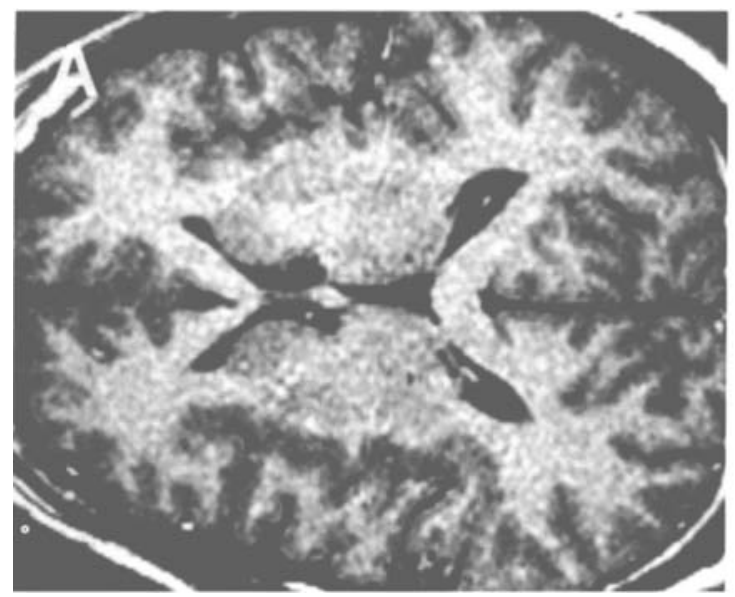

the influence of the prior term gives way to the noise term, and the restoration takes on the appearance of the ML solution as $\tau \rightarrow 0$. The prior energy formulations for $\mathrm{T} 1$ and $\mathrm{T} 2$ are isomorphic to that of PD.

Summing the prior energy contributions results in a function

$$
\mathrm{H}_{\mathrm{P}}=\sum_{\mathrm{i}}\left(\mathrm{E}_{\mathrm{PD}, \mathrm{i}}+\mathrm{E}_{\mathrm{T} 1, \mathrm{i}}+\mathrm{E}_{\mathrm{T} 2, \mathrm{i}}\right)
$$

which represents a degree of smoothness within a local region of the $\mathrm{PD}, \mathrm{T} 1$, and $\mathrm{T} 2$ images. Thus, if we can find values for $\mathrm{PD}, \mathrm{T} 1$, and $\mathrm{T} 2$ that minimize a cost function

$$
\mathrm{H}=\mathrm{H}_{\mathrm{N}}+\mathrm{H}_{\mathrm{p}}
$$

at each value of $\tau$, starting at a high value for $\tau$, and halting the process when $\tau$ is small enough,

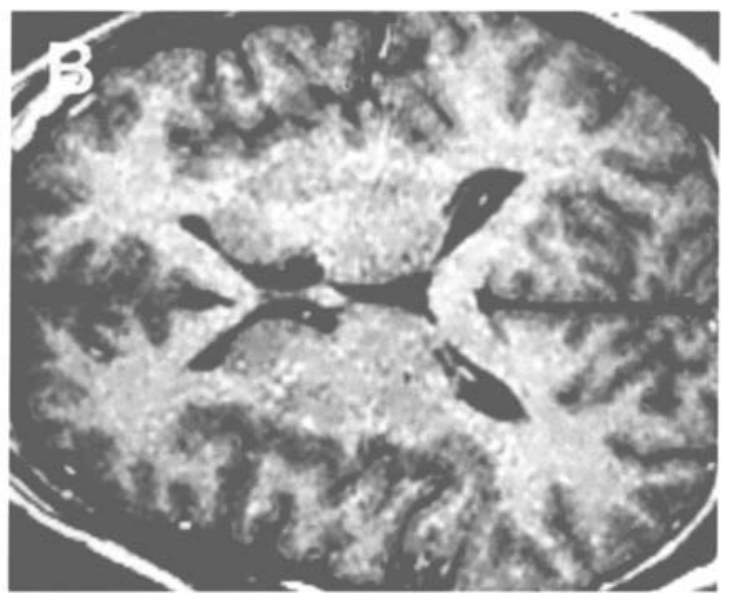

Fig 2. TE, 17; TR, 500. Left, Data; Right, MAP restoration. Three millimeter slice with 3-mm spacing. 

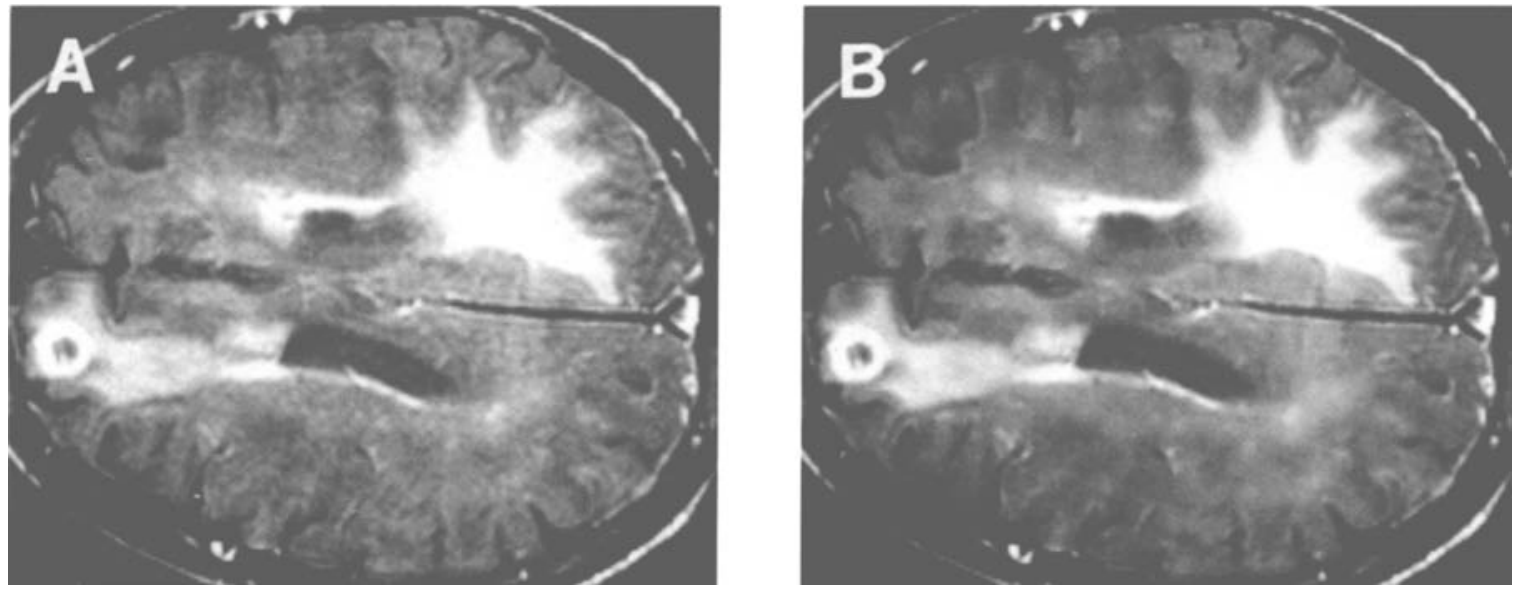

Fig 3. TE, 32; TR, 3,000. Left. Data; Right, MAP restoration. Five millimeter slice with 1-mm spacing. Gadolinium administered before study.

we will obtain a restoration that retains fidelity to the data, preserves step edges, and suppresses noise.

\section{MATERIALS AND METHODS}

We acquired several sets of three images each from a $\mathrm{GE}$ Signa 5.2 scanner with a 1.5 Tesla magnet using spin-echo mode, acquiring one Tl-weighted study and one PD/T2weighted multi-echo study for a total of three images per set. TE and TR values set for the presumably healthy brain in the left half of Figs 1 and 2 were 90 and 3,000 and 17 and 500 , respectively. The data corresponding to the TE and TR values of 30 and 3,000 , respectively, are not shown. This particular image set was obtained from a study using $3-\mathrm{mm}$ gaps between adjacent $3-\mathrm{mm}$ slices with a field of view of $22 \mathrm{~cm}$.

The brain images that show pathology in the left half of Figs 3 and 4 were obtained after administration of gadolinium. The TE and TR values were set at 32 and 3,000 and 90

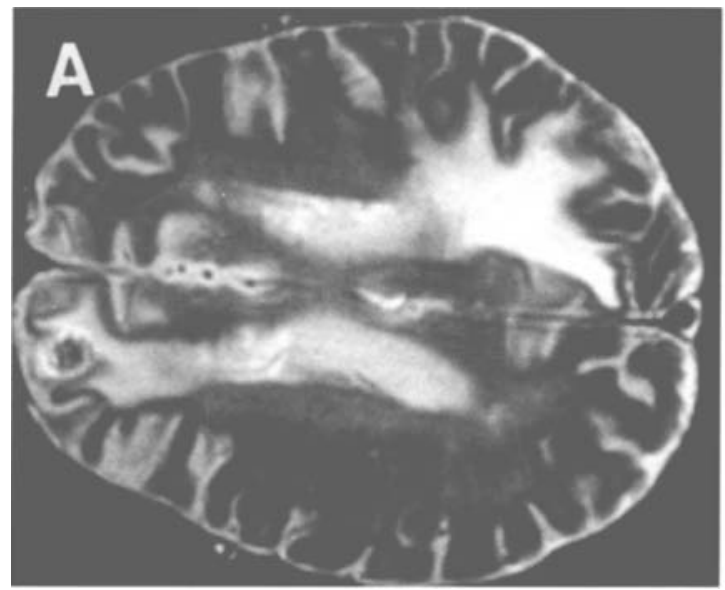

and 3,000, respectively. The data corresponding to TE and TR values of 17 and 417, respectively, are not shown. This study used $1-\mathrm{mm}$ gaps between $5-\mathrm{mm}$ slices. The field of view was set at $22 \mathrm{~cm}$.

The noisy images of a presumably healthy brain in the left half of Figs 5 and 6 used TE and TR settings of 90 and 900 and 90 and 1,800 , respectively. The data corresponding to TE and TR values of 30 and 900 , respectively, are not shown. This study used 0 -mm gaps between 3 -mm slices. Again, the field of view was fixed at $22 \mathrm{~cm}$.

All of these data images were processed using both the ML and MAP algorithms. The $H$ of equation 6 was minimized with respect to $\mathrm{PD}, \mathrm{T} 1$, and $\mathrm{T} 2$ using gradient descent. See Garnier et al ${ }^{7.8}$ for details. Because only three data images were used during each restoration, the ML restorations showed no improvement over the data. Values for the $\sigma_{c}$ in the noise term were hand estimated from a region of tissue in each acquired image that appeared to have an underlying piecewise-constant value. Values for $\sigma_{\mathrm{PD}}, \sigma_{\mathrm{T} 1}$, and $\sigma_{\mathrm{T} 2}$ were similarly determined from a region of

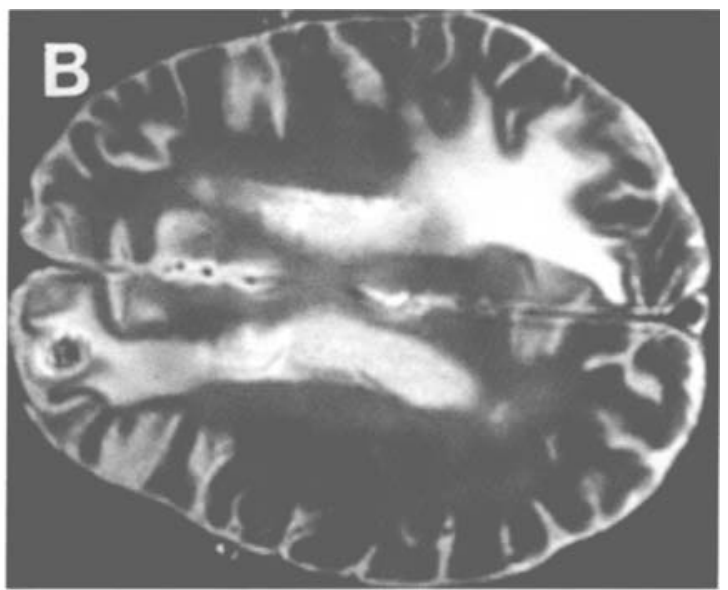

Fig 4. TE, 90; TR, 3,000. Left, Data; Right, MAP restoration. Five millimeter slice with 1-mm spacing. Gadolinium administered prior to study. 

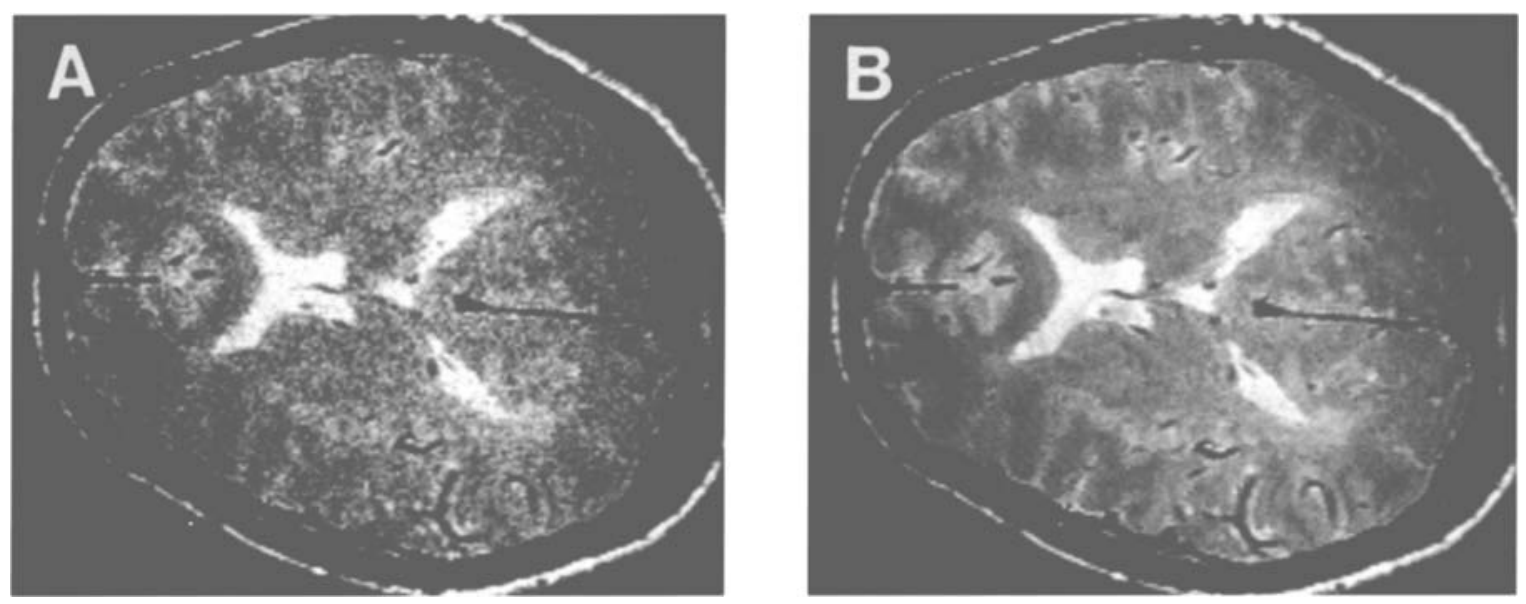

Fig 5. TE, 90; TR, 900. Left, Data; Right, MAP restoration. Three millimeter slice with 0-mm spacing.

tissue in each ML-restored basis image that also appeared to have an underlying piecewise-constant value.

\section{RESULTS}

The images in the right half of Figures 1 through 6 are typical synthetic restorations produced by the MAP algorithm. The $\sigma_{\mathrm{c}}, \sigma_{\mathrm{PD}}$, $\sigma_{\mathrm{T} 1}$, and $\sigma_{\mathrm{T} 2}$ were all determined from whitematter regions. We also used $\sigma_{\mathrm{PD}}, \sigma_{\mathrm{T} 1}$ and $\sigma_{\mathrm{T} 2}$ determined as a fraction of the mean value from their corresponding white-matter signals in an effort to model an equivalent degree-of-smoothness across all basis images, but found the restorations to be visually equivalent to those presented here. The intent of this work was to show the restoration ability of our approach; it was not our primary intent to develop a fast application tool. We wish to note that we have

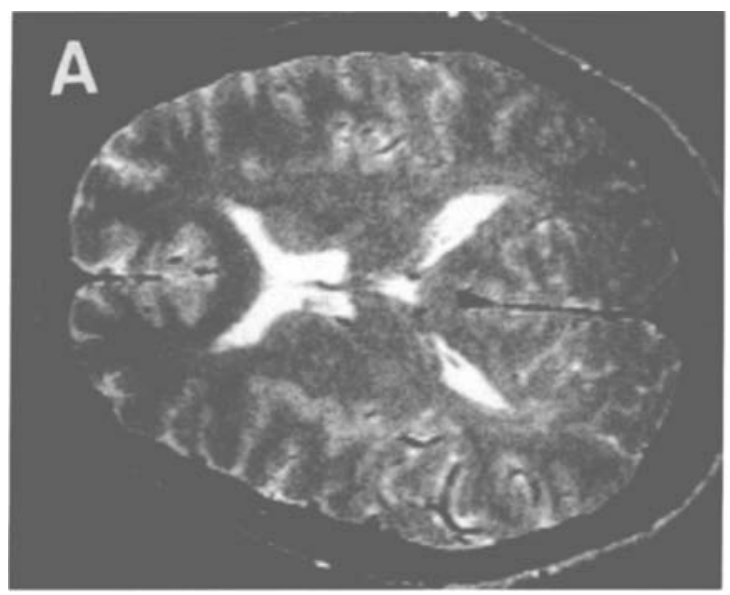

used the simplest possible gradient descent scheme, which a modern workstation would implement in $\sim 30$ minutes. A factor of 5 or 10 could be gained by using more efficient numerical procedures, ${ }^{6.9}$ and similar algorithms have been accelerated by a factor of 30 to 40 using parallel processors. ${ }^{10}$

\section{CONCLUSIONS AND FUTURE WORK}

We have shown two novel results: (1) A method of computing PD, T1, and T2 from any set of images with distinct TE and TR values. (All previous reported work chose special values for TE or TR, which simplified the coupled spin equations), and (2) a method for removing noise (while still preserving the sharpness of edges) from these images, which seems to be

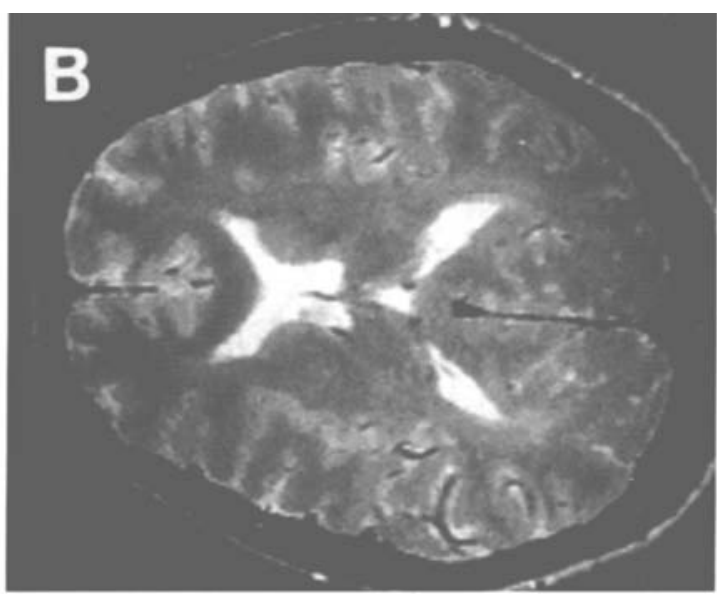

Fig 6. TE, 90; TR, 1,800. Left, Data; Right, MAP restoration. Three millimeter slice with 0-mm spacing. 
superior to any method previously reported. ${ }^{7}$ When used in the spin-echo application, the method suffers from the requirement that the patient must remain motionless between the multiple studies that use dissimilar TR values. Although shown using readily available spinecho images, the method is readily adaptable to other MRI imaging modes by simply changing the form of the physical relation equation.

It may be argued that in conventional spin echo images, noise-like artifacts arise primarily from metabolic motion and are not (for the most part) from true random noise. However, in echo planar imaging, acquisition times are so short that motion artifacts are dramatically reduced and to some extent replaced by random noise (with which this algorithm performs best). In imaging using an magnetization transfer cross-relaxation contrast preparatory pulse, the formation equation has a form similar to equation 1 , and this method should be applicable to removing noise from such images. Investigations are under way.

\section{ACKNOWLEDGMENT}

We thank W.H. Hinson and C.A. Hamilton at the Bowman-Gray School of Medicine, Wake Forest University, for providing data images.

\section{APPENDIX}

The formulation of Bayes' rule can be found in Fukumaga. ${ }^{11}$ Geman and Geman ${ }^{12}$ rigorously and effectively applied Bayesian techniques to the restoration of images that were remodeled as Markov random fields. Marroquin, et al ${ }^{13}$ applied regularization techniques using prior knowledge. Hiriyannaiah et al ${ }^{14}$ used deterministic approximations for piecewise-constant restorations. Bilbro and Snyder ${ }^{15}$ later applied more sophisticated priors.

Let $G$ be a measured vector set of images, $\mathrm{G}=\left\{\mathrm{g}_{\mathrm{c}}\right\}_{\mathrm{c}=1, \mathrm{~d}}$ and $\mathrm{g}_{\mathrm{c}}=\left[\mathrm{g}_{\mathrm{c}, \mathrm{i}}\right]_{\mathrm{i}=1, \mathrm{n}}$ where $\mathrm{d}$ is the number of channels in the vector set, and where $\mathrm{g}_{\mathrm{c}, \mathrm{i}}$ represents the $c$ th channel value associated with the $i$ th pixel. Using similar notation, let $S(F)$ represent the undegraded ideal images as a deterministic function of $F$, where $F$ are the undegraded ideal basis images, and let $\mathrm{N}$ represent additive noise such that $\mathrm{G}=\mathrm{S}+\mathrm{N}$. Note that $F=\left\{f_{\psi}\right\}_{\psi=1, p}$ and $f_{\psi}=\left[f_{\psi, i}\right]_{i=1, n}$ where $p$ is the number of basis images in the vector set, and where $f_{u, i}$ represents the value associated with the $i$ th pixel of the $\psi$ th basis image.

In Bayesian restoration, the most acceptable result is the estimate with the highest probability of occurrence. Let $\hat{F}$ be an estimate of $F$. Bayes' rule gives the posterior distribution ${ }^{11,12}$ of $\hat{F}$ given the data $G$ as

$$
P(\hat{F} \mid G)=\frac{P(G \mid \hat{F}) P(\hat{F})}{P(G)} .
$$

We refer to $P(G / \hat{F})$ as the "noise term" and it describes the noise distribution. $P(\hat{F})$ is called the "prior term" and it describes the a priori distribution that can be chosen using a priori knowledge about $F, P(G)$ is constant and independent of $\hat{F}$, so to maximize the posterior distribution, we need only maximize $P(G / \hat{F}) \times$ $\mathrm{P}(\hat{\mathbf{F}})$. The ML approach does not incorporate a prior model, hence the restoration is based solely on the noise term. For the ML case, the noise that has corrupted $\mathrm{S}(\mathrm{F})$ is modeled as stationary, signal-independent additive Gaussian noise, hence $P(G / F)$ is given by

$$
P(G \mid \hat{F})=P(N) \equiv \frac{\exp \left(-1 / 2 N^{T} C_{G}^{-1} N\right)}{(2 \pi)^{n d / 2}\left|C_{G}\right|^{1 / 2}}
$$

where $\mathrm{C}_{\mathrm{G}}$ denotes the covariance matrix of noise $N$, and $\left|C_{G}\right|$ denotes the determinant of $\mathrm{C}_{\mathrm{G}}$. Taking the negative $\log$ of $\mathrm{P}(\mathrm{G} / \hat{\mathrm{F}})$ and harmlessly ignoring constant contributions, a "noise cost" of a cost function is obtained:

$$
\mathrm{H}_{\mathrm{N}}(\hat{\mathrm{F}}, \mathrm{G})=\sum_{\mathrm{c}} \frac{1}{2 \sigma_{\mathrm{c}}^{2}} \sum_{\mathrm{i}}\left(\hat{\mathrm{s}}_{\mathrm{c}, \mathrm{i}}(\hat{\mathrm{F}})-\mathrm{g}_{\mathrm{c}, \mathrm{i}}\right)^{2},
$$

for isotropic uncorrelated noise.

Smoothing requires the use of an additional term in the cost function, the prior term, this term, $\mathrm{P}(\hat{\mathrm{F}})$, depends only on $\hat{\mathrm{F}}$, and should reflect some prior knowledge of the nature of $\mathrm{F}$. For the case of MR images, the basis images should appear to be locally homogeneous in some important way. Because it is frequently used in similar restoration problems, ${ }^{12}$ an exponential form is chosen for $P(\hat{F})$ :

$$
P(\hat{F})=\frac{\exp \left(-\mathrm{H}_{\mathrm{p}}(\hat{\mathrm{F}})\right)}{\mathrm{Z}_{\mathrm{p}}}
$$

where $Z_{p}$ normalizes $\mathrm{P}(\hat{\mathrm{F}}) . \mathrm{H}_{\mathrm{p}}(\hat{\mathrm{F}})$ in this in- 
stance is used to measure and regulate the local homogeneity of some characteristic of the basis images. Taking the negative $\log$ of $\mathrm{P}(\hat{\mathrm{F}})$ results in a "prior cost" for a cost function.

\section{REFERENCES}

1. Liu J, Nieminen AOK, Koenig JL: Calculation of $T_{1}$, $T_{2}$, and proton spin density images in nuclear magnetic resonance imaging. J Magn Reson Imaging 85:95-110, 1989

2. Ortendahl DA, Hylton N, Kaufman L, et al: Analytic tools for magnetic resonance imaging. Radiology 153:479488, 1984

3. Lee JN, Riederer SJ, Bobman SA, et al: Instrumentation for rapid MR image synthesis. Magn Reson Med 3:33-43, 1986

4. Bobman SA, Riederer SJ, Lee JN, et al: Pulse sequence extrapolation with MR image synthesis. Radiology 159:253-258, 1986

5. MacFall JR, Riederer SJ, Wang HZ: An analysis of noise propagation in computed $\mathrm{T}_{2}$, pseudodensity, and synthetic spin-echo images. Med Phys 13:285-292, 1986

6. Bilbro GL, Snyder WE, Garnier SJ, et al: Mean field annealing: A formalism for constructing GNC-like algorithms. IEEE Trans Neural Networks 3:131-138, 1992

7. Garnier SJ, Bilbro GL, Gault W, et al: Magnetic resonance image restoration: J Math Imaging Vision (in press)

8. Garnier SJ: Recovering resolution and reducing noise in basis images via optimization methods using physical models. Doctoral thesis, North Carolina State University, Raleigh, NC, 1994

9. Blake A, Zisserman A: Visual Reconstruction. Cambridge, MA, MIT, 1987

10. Bilbro GL, Snyder WE, Mann RC: Mean-field approximation minimizes relative entropy. J Opt Soc Am [A] 8:290-294, 1991

11. Fukunaga K: Introduction to Statistical Pattern Recognition. San Diego, CA, Academic, 1972

12. Geman D, Geman S: Stochastic relaxation, Gibbs distributions, and the Bayesian restoration of images. IEEE Trans Pattern Anal Machine Intelligence 6:721-741, 1984

13. Marroquin, Mitter S, Poggio T: Probabalistic solution of ill-posed problems in computational vision. J Am Stat Assoc 82:76-89, 1987

14. Hiriyannaiah H, Bilbro GL, Snyder WE, et al: Restoration of piecewise constant images via mean field annealing. J Opt Soc Am [A] 6:1901-1912, 1989

15. Bilbro GL, Snyder WE: Applying mean field annealing to image noise removal. J Neural Network Computing $1: 5-17,1990$ 